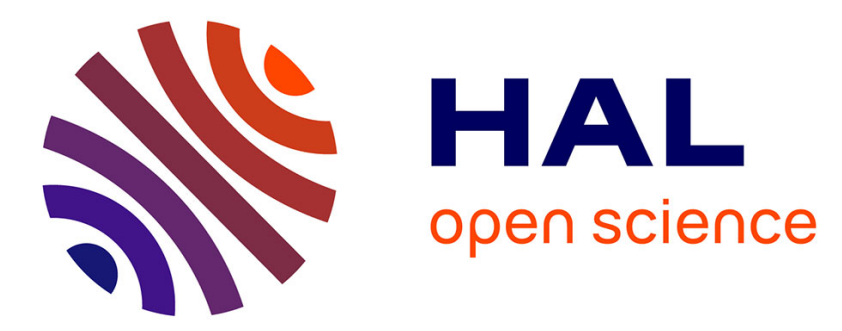

\title{
Weiss-Weinstein bound for an abrupt unknown frequency change
}

Lucien Bacharach, Mohammed Nabil El Korso, Alexandre Renaux

\section{To cite this version:}

Lucien Bacharach, Mohammed Nabil El Korso, Alexandre Renaux. Weiss-Weinstein bound for an abrupt unknown frequency change. 2016 IEEE Statistical Signal Processing Workshop (SSP), Jun 2016, Palma de Majorque, Spain. 10.1109/ssp.2016.7551787 . hal-01346610

\section{HAL Id: hal-01346610 \\ https://hal-centralesupelec.archives-ouvertes.fr/hal-01346610}

Submitted on 19 Jul 2016

HAL is a multi-disciplinary open access archive for the deposit and dissemination of scientific research documents, whether they are published or not. The documents may come from teaching and research institutions in France or abroad, or from public or private research centers.
L'archive ouverte pluridisciplinaire HAL, est destinée au dépôt et à la diffusion de documents scientifiques de niveau recherche, publiés ou non, émanant des établissements d'enseignement et de recherche français ou étrangers, des laboratoires publics ou privés. 


\title{
WEISS-WEINSTEIN BOUND FOR AN ABRUPT UNKNOWN FREQUENCY CHANGE
}

\author{
Lucien Bacharach*, Mohammed Nabil El Korso ${ }^{\dagger}$ and Alexandre Renaux* \\ * Université Paris-Sud/L2S, 3 rue Joliot-Curie, 91192 Gif-sur-Yvette \\ ${ }^{\dagger}$ Université Paris-Ouest/LEME, 50 rue de Sèvres, 92410 Ville d'Avray
}

\begin{abstract}
In this paper, we derive analytical expressions of the WeissWeinstein bound (WWB) in the context of observations whose frequency abruptly changes at an unknown time instant. Since both frequencies before and after the change are assumed to be unknown as well, it is appropriate to consider the multiparameter version of the WWB. Furthermore, numerical simulations are provided in order to illustrate the tightness of the proposed bound expressions regarding to the estimates errors.
\end{abstract}

Index Terms - Weiss-Weinstein bound, change-point, frequency estimation, MSE bayesian lower bound

\section{INTRODUCTION}

In many practical applications, abrupt changes, refered to as change-points, may occur in the distribution of the signal of interest, e.g., in quality control, in speech processing, as well as in navigation system monitoring [1]. Such problem is also encountered in radar signal processing, and more specifically in SAR image edge detection [2], in clutter map segmentation [3] or in the detection of target signals in clutter [4]. Most often, the time instants at which the changes occur are unknown and must be estimated jointly with the parameters of interest that change from one change-point to another.

In such scenario, change-point estimation has been widely studied in the last five decades. A plethora of algorithms have been proposed, among which the maximum likelihood based schemes received a particular attention [5, 6, 7]. Its optimality properties led to important studies of its asymptotic distribution. First, in [5], Hinlkey derived the exact asymptotic distribution of the maximum likelihood estimate (MLE), and proposed in the same paper a computationally tractable approximation of the latter. Fotopoulos and Jandhyala later worked on deriving exact computable forms of the asymptotic distribution of the MLE, in the cases of exponentially distributed and Gaussian random processes [6,7]. The knowledge of the distribution of the MLE, which is highly informative, seems unfortunately impossible to obtain in non asymp-

This work has been partially supported by the iCODE institute, research project of the IDEX Paris-Saclay, by the MAGELLAN project (ANR-14CE23-0004-01) and by the TITAN Mastodons project of CNRS. totic context. At least, as far as we know, such result does not exist in the literature. To overcome this difficulty, an alternative is to study the so-called lower bounds on the variance of the change-point estimation problem. One of the most commonly used lower bound on the mean squarre error (MSE), is the Cramér-Rao bound (CRB) due to its easy derivation and tightness in the asymptotic region $[8,9]$. Nevertheless, the CRB is not tight in the non-asymptotic region and more importantly, it requires strong regularity conditions as the smoothness of the log likelihood fonction which is not satisfied in our context since the change-point parameter is discrete. A first alternative was to regularize the problem by approximating the signal by a smoother one $[10,11]$. A second alternative was to consider a lower bound on the MSE that does not require the smoothness of the log-likelihood function. This has been first done using the Chapman-Robbins bound for a single change-point in [12], and then extended to multiple changes in [13], both with known parameters of interest before and after the change-point(s). The results in both cases are not satisfying since the resulting bounds are not tight. This drawback led us to consider the problem from a Bayesian point of view, for which we recently derived the Weiss-Weinstein bound (WWB) for a single change-point and known parameters of interest [14].

In this paper, we extend [14] to the case of complex observation vector with unknown (changing) parameters of interest. Specifically, we consider a widely used model in the literature, namely the complex sinusoidal signal, where the unknown parameters of interest (frequencies) are suject to a change at an unknown time instant. We chose cisoid model for sake of clarity, nevertheless, it is encountered in several radar area or can be easily extended to specific radar applications involving sophisticated model based on the cisoid. One can cite target detection in abruptly non-stationary Dopplerspread clutter $[15,4]$. The aim is to analyze the estimation performance on both the frequencies before and after the change jointly with the time instant at which the change occurs. It is known that the problem of frequency estimation is non-linear and gives rise to a threshold phenomenon under which the estimation performance is highly degraded [16]. Consequently, the use of the WWB is interesting since the breakdown point is also rendered by this bound, unlike the CRB [17]. Finally, this study allows us to have a glance at 
how the estimation behavior is influenced by the joint estimation of these two types of parameters, namely the discrete change-point as well as the continuous frequencies parameters.

\section{PROBLEM FORMULATION}

Let us consider the complex sinusoidal model for frequency change-point scenario given by

$$
\begin{cases}y(n)=\mathrm{e}^{\mathrm{i} 2 \pi f_{1} n}+w(n) & \text { for } n=1, \ldots, \tau \\ y(n)=\mathrm{e}^{\mathrm{i} 2 \pi f_{2} n}+w(n) & \text { for } n=\tau+1, \ldots, N\end{cases}
$$

where $y(n)$ denotes the observation at the time instant $n, f_{1}$ and $f_{2}$ are unknown (normalized) frequencies, $\tau$ is the unknown change-point, and $w(n)$ is a zero mean, complex, circular, Gaussian, i.i.d. noise with variance $\sigma^{2}$. Model (1) can be seen as a simplified model of radars for target detection in abruptly non-stationary Doppler-spread clutter [15, 4].

The whole observation can be gathered in the vector $\mathbf{y} \triangleq(y(1), \ldots, y(N))^{T} \in \Omega$, whose mean is denoted by $\mathbf{s}_{\tau}\left(f_{1}, f_{2}\right) \triangleq\left(\mathrm{e}^{\mathrm{i} 2 \pi f_{1}}, \ldots, \mathrm{e}^{\mathrm{i} 2 \pi f_{1} \tau}, \mathrm{e}^{\mathrm{i} 2 \pi f_{2}(\tau+1)}, \ldots, \mathrm{e}^{\mathrm{i} 2 \pi f_{2} N}\right)^{T}$ and the proper noise vector by $\mathbf{w} \triangleq(w(1), \ldots, w(N))^{T} \sim$ $\mathbb{C} \mathcal{N}\left(\mathbf{0}, \sigma^{2} \mathbf{I}_{N}\right)$. Consequently, (1) can be rewritten as

$$
\mathbf{y}=\mathbf{s}_{\tau}\left(f_{1}, f_{2}\right)+\mathbf{w} .
$$

Following a Bayesian approach, we assume a uniform $a$ priori for the normalized frequencies, i.e., $f_{i} \sim \mathcal{U}_{\left[-\frac{1}{2}, \frac{1}{2}\right]}$, $i=1,2$, and that $\tau$ follows a discrete uniform distribution between 1 and $N-1$, i.e., $\tau \sim \mathcal{U}_{\{1, \ldots, N-1\}}$. The unknown parameter vector is denoted by $\boldsymbol{\theta}=\left(f_{1}, f_{2}, \tau\right)^{T} \in \Theta$, in which $\Theta=\mathbb{R}^{2} \times \mathbb{N}$. Since every component of $\boldsymbol{\theta}$ is independent one from another, the joint prior pdf can be written as

$$
p\left(f_{1}, f_{2}, \tau=k\right)=I_{\left[-\frac{1}{2}, \frac{1}{2}\right]}\left(f_{1}\right) I_{\left[-\frac{1}{2}, \frac{1}{2}\right]}\left(f_{2}\right) \frac{I_{\{1, \ldots, N-1\}}(k)}{N-1}
$$

where $I_{\mathcal{A}}(x)$ denotes the indicator function w.r.t. the set $\mathcal{A}$, i.e., it equals 1 if $x \in \mathcal{A}$ and 0 otherwise.

\section{WWB DEFINITION AND DERIVATION}

\subsection{Background on the WWB}

The multiparameter form of the WWB [18] satisfies the following matrix inequality for any Bayesian estimator $\widehat{\boldsymbol{\theta}}(\mathbf{y})$ of $\theta$ :

$$
\mathbb{E}_{\mathbf{y}, \boldsymbol{\theta}}\left\{[\widehat{\boldsymbol{\theta}}(\mathbf{y})-\boldsymbol{\theta}][\widehat{\boldsymbol{\theta}}(\mathbf{y})-\boldsymbol{\theta}]^{T}\right\} \succeq \mathbf{H G}^{-1} \mathbf{H}^{T}
$$

in which $\mathbf{A} \succeq \mathbf{B}$ means $\mathbf{A}-\mathbf{B}$ is a nonnegative matrix, $\mathbf{H}=$ $\left[\boldsymbol{\theta}_{1}-\boldsymbol{\theta}, \boldsymbol{\theta}_{2}-\boldsymbol{\theta}, \boldsymbol{\theta}_{3}-\boldsymbol{\theta}\right]$ is a function of the so-called testpoints $\left\{\boldsymbol{\theta}_{1}, \boldsymbol{\theta}_{2}, \boldsymbol{\theta}_{3}\right\}$ which can be chosen by the user as long as each $\boldsymbol{\theta}_{q} \in \Theta$. We define $\mathbf{h}_{q}=\boldsymbol{\theta}_{q}-\boldsymbol{\theta}$ and $\mathbf{H}$ becomes $\mathbf{H}=$ $\left[\mathbf{h}_{1}, \mathbf{h}_{2}, \mathbf{h}_{3}\right]$. The $3 \times 3$ matrix $\mathbf{G}$ is defined by its $(i, j)$-th element as

$$
G_{i, j}=\frac{\mathbb{E}_{\mathbf{y}, \boldsymbol{\theta}}\left\{\begin{array}{c}
{\left[L^{s_{i}}\left(\mathbf{y}, \boldsymbol{\theta}+\mathbf{h}_{i}, \boldsymbol{\theta}\right)-L^{1-s_{i}}\left(\mathbf{y}, \boldsymbol{\theta}-\mathbf{h}_{i}, \boldsymbol{\theta}\right)\right]} \\
\left.\times\left[L^{s_{j}}\left(\mathbf{y}, \boldsymbol{\theta}+\mathbf{h}_{j}, \boldsymbol{\theta}\right)-L^{1-s_{j}}\left(\mathbf{y}, \boldsymbol{\theta}-\mathbf{h}_{j}, \boldsymbol{\theta}\right)\right]\right\}
\end{array}\right.}{\mathbb{E}_{\mathbf{y}, \boldsymbol{\theta}}\left\{L^{s_{i}}\left(\mathbf{y}, \boldsymbol{\theta}+\mathbf{h}_{i}, \boldsymbol{\theta}\right)\right\} \mathbb{E}_{\mathbf{y}, \boldsymbol{\theta}}\left\{L^{s_{j}}\left(\mathbf{y}, \boldsymbol{\theta}+\mathbf{h}_{j}, \boldsymbol{\theta}\right)\right\}}
$$

where $L(\mathbf{y}, \boldsymbol{\alpha}, \boldsymbol{\beta})=\frac{p(\mathbf{y}, \boldsymbol{\alpha})}{p(\mathbf{y}, \boldsymbol{\beta})}$. The inequality (4) holds for all combinations of $\mathbf{h}_{q}$ and $0<s_{q}<1$ such that $\mathbf{G}$ is invertible. Then, the WWB is obtained by maximizing the right side of (4) w.r.t. the degrees of freedom $\mathbf{h}_{q}$ and $s_{q}$. To reduce the computational cost of this maximization procedure, we first assume one test point per parameter, i.e., $\mathbf{h}_{q}$ has only one nonzero element $h_{q}$, leading to $\mathbf{H}=\operatorname{diag}\left(h_{1}, h_{2}, h_{3}\right)$. Second, it often has been noticed numerically that choosing $s_{q}=\frac{1}{2} \forall q$ leads to the maximum bound, thus we make this choice here and perform the maximization only with respect to $h_{1}, h_{2}$ and $h_{3}$. Consequently, (5) can be rewritten as

$$
G_{i, j}=\frac{\zeta\left(\mathbf{h}_{i}, \mathbf{h}_{j}\right)+\zeta\left(-\mathbf{h}_{i},-\mathbf{h}_{j}\right)-\zeta\left(\mathbf{h}_{i},-\mathbf{h}_{j}\right)-\zeta\left(-\mathbf{h}_{i}, \mathbf{h}_{j}\right)}{\zeta\left(\mathbf{h}_{i}, \mathbf{0}\right) \zeta\left(\mathbf{h}_{j}, \mathbf{0}\right)}
$$

where

$$
\zeta\left(\mathbf{h}_{i}, \mathbf{h}_{j}\right)=\iint_{\Theta} \sqrt{p\left(\mathbf{y}, \boldsymbol{\theta}+\mathbf{h}_{i}\right) p\left(\mathbf{y}, \boldsymbol{\theta}+\mathbf{h}_{j}\right)} \mathrm{d} \mathbf{y} \mathrm{d} \boldsymbol{\theta}
$$

in which the notation $\int_{\Theta} \cdot \mathrm{d} \boldsymbol{\theta}$ stands for $\iint_{\mathbb{R}} \sum_{\mathbb{N}} \cdot \mathrm{d} f_{1} \mathrm{~d} f_{2}$.

\subsection{Derivation of the WWB}

\subsubsection{Derivation of the diagonal terms $G_{1,1}$ and $G_{2,2}$}

We start by deriving $G_{1,1}$, relative to frequency $f_{1}$, from which we will deduce $G_{2,2}$ for frequency $f_{2}$. First,

$\zeta\left(\mathbf{h}_{1}, \mathbf{0}\right)=\int_{\Theta}\left[\int_{\Omega} \sqrt{p\left(\mathbf{y} \mid \boldsymbol{\theta}+\mathbf{h}_{1}\right) p(\mathbf{y} \mid \boldsymbol{\theta})} \mathrm{d} \mathbf{y}\right] \sqrt{p\left(\boldsymbol{\theta}+\mathbf{h}_{1}\right) p(\boldsymbol{\theta})} \mathrm{d} \boldsymbol{\theta}$

where $p(\boldsymbol{\theta})$ is given by (3) and $p\left(\boldsymbol{\theta}+\mathbf{h}_{1}\right)$ is determined accordingly:

$p\left(\boldsymbol{\theta}+\mathbf{h}_{1}\right)=I_{\left[-\frac{1}{2}-h_{1}, \frac{1}{2}-h_{1}\right]}\left(f_{1}\right) I_{\left[-\frac{1}{2}, \frac{1}{2}\right]}\left(f_{2}\right) \frac{I_{\{1, \ldots, N-1\}}(k)}{N-1}$

Consequently,

$$
\begin{aligned}
\sqrt{p\left(\boldsymbol{\theta}+\mathbf{h}_{1}\right) p(\boldsymbol{\theta})}=I_{\left[-\frac{1}{2}+\left(-h_{1}\right)^{+}, \frac{1}{2}-\left(h_{1}\right)^{+}\right]}\left(f_{1}\right) & I_{\left[-\frac{1}{2}, \frac{1}{2}\right]}\left(f_{2}\right) \\
& \times \frac{I_{\{1, \ldots, N-1\}}(k)}{N-1}
\end{aligned}
$$

where for any real parameter, $(x)^{+} \triangleq \max (x, 0)$.

On the other hand, from the i.i.d. assumption we have

$$
p(\mathbf{y} \mid \boldsymbol{\theta}) \triangleq p\left(\mathbf{y} \mid f_{1}, f_{2}, \tau=k\right)=\prod_{n=1}^{k} p\left(y(n) \mid f_{1}\right) \prod_{n=k+1}^{N} p\left(y(n) \mid f_{2}\right)
$$


and $p\left(\mathbf{y} \mid \boldsymbol{\theta}+\mathbf{h}_{1}\right)$ is modified accordingly. This leads to:

$$
\begin{gathered}
\sqrt{p\left(\mathbf{y} \mid \boldsymbol{\theta}+\mathbf{h}_{1}\right) p(\mathbf{y} \mid \boldsymbol{\theta})}=\frac{1}{\left(\pi \sigma^{2}\right)^{N}} \exp \left\{-\frac{1}{\sigma^{2}}\left(\mathbf{y}-\mathbf{m}_{\mathbf{s}}\right)^{H}\left(\mathbf{y}-\mathbf{m}_{\mathbf{s}}\right)\right\} \\
\times \exp \left\{-\frac{\left(N-\operatorname{Re}\left(\mathbf{s}_{k}\left(f_{1}+h_{1}, f_{2}\right)^{H} \mathbf{s}_{k}\left(f_{1}, f_{2}\right)\right)\right)}{2 \sigma^{2}}\right\}
\end{gathered}
$$

where $\mathbf{m}_{\mathbf{s}}=\frac{1}{2}\left(\mathbf{s}_{k}\left(f_{1}+h_{1}, f_{2}\right)+\mathbf{s}_{k}\left(f_{1}, f_{2}\right)\right)$. Then, the integration over $\Omega$ with respect to $\mathbf{y}$ gives:

$$
\begin{aligned}
& \int_{\Omega} \sqrt{p\left(\mathbf{y} \mid \boldsymbol{\theta}+\mathbf{h}_{1}\right) p(\mathbf{y} \mid \boldsymbol{\theta})} \mathrm{d} \mathbf{y}= \\
& \exp \left\{-\frac{\left(N-\operatorname{Re}\left(\mathbf{s}_{k}\left(f_{1}+h_{1}, f_{2}\right)^{H} \mathbf{s}_{k}\left(f_{1}, f_{2}\right)\right)\right)}{2 \sigma^{2}}\right\}
\end{aligned}
$$

More explicitely, since

$$
\begin{aligned}
\mathbf{s}_{k}\left(f_{1}+h_{1}, f_{2}\right)^{H} \mathbf{s}_{k}\left(f_{1}, f_{2}\right) & =\sum_{n=1}^{k} \mathrm{e}^{-\mathrm{i} 2 \pi h_{1} n}+N-k \\
& =\mathrm{e}^{-\mathrm{i} \pi h_{1}(k+1)} \frac{\sin \left(\pi h_{1} k\right)}{\sin \left(\pi h_{1}\right)}+N-k,
\end{aligned}
$$

the left side of (13) does not depend on $f_{1}$ and $f_{2}$. Finally, by substituting (13) and (10) into (8) and by integrating out $f_{1}$ and $f_{2}$ and summing over $\mathbb{N}$, we obtain

$\zeta\left(\mathbf{h}_{1}, \mathbf{0}\right)=\frac{1-\left|h_{1}\right|}{N-1} \sum_{k=1}^{N-1} \exp \left\{-\frac{1}{4 \sigma^{2}}\left(2 k+1-\frac{\sin \left(\pi h_{1}(2 k+1)\right)}{\sin \left(\pi h_{1}\right)}\right)\right\}$

which concludes the derivation of the denominator terms of (6). For the numerator, first, we notice that

$$
\zeta\left(\mathbf{h}_{1}, \mathbf{h}_{1}\right)=\zeta\left(-\mathbf{h}_{1},-\mathbf{h}_{1}\right)=\iint_{\Omega} p\left(\mathbf{y}, \boldsymbol{\theta} \pm \mathbf{h}_{1}\right) \mathrm{d} \mathbf{y} \mathrm{d} \boldsymbol{\theta}=1 .
$$

The other terms of the numerator of (6) can be derived as follows:

$$
\zeta\left(\mathbf{h}_{1},-\mathbf{h}_{1}\right)=\zeta\left(-\mathbf{h}_{1}, \mathbf{h}_{1}\right)=\zeta\left(2 \mathbf{h}_{1}, \mathbf{0}\right)
$$

using a proper variable change. Finally, by substituting (16) and (17) into (6), we obtain

$$
G_{1,1}=\frac{2\left(1-\zeta\left(2 \mathbf{h}_{1}, \mathbf{0}\right)\right)}{\zeta^{2}\left(\mathbf{h}_{1}, \mathbf{0}\right)}
$$

where $\zeta\left(\mathbf{h}_{1}, \mathbf{0}\right)$ and $\zeta\left(2 \mathbf{h}_{1}, \mathbf{0}\right)$ are given according to (15).

Following the same methodology, we obtain

$$
G_{2,2}=\frac{2\left(1-\zeta\left(2 \mathbf{h}_{2}, \mathbf{0}\right)\right)}{\zeta^{2}\left(\mathbf{h}_{2}, \mathbf{0}\right)}
$$

in which

$$
\begin{aligned}
& \zeta\left(\mathbf{h}_{2}, \mathbf{0}\right)= \frac{1-\left|h_{2}\right|}{N-1} \sum_{k=1}^{N-1} \exp \left\{-\frac{1}{2 \sigma^{2}}(N-k-\right. \\
&\left.\left.\cos \left(\pi h_{2}(N+k+1)\right) \frac{\sin \left(\pi h_{2}(N-k)\right)}{\sin \left(\pi h_{2}\right)}\right)\right\} .
\end{aligned}
$$

\subsubsection{Derivation of the diagonal term $G_{3,3}$}

First, (10) becomes

$$
\begin{aligned}
\sqrt{p\left(\boldsymbol{\theta}+\mathbf{h}_{3}\right) p(\boldsymbol{\theta})}=I_{\left[-\frac{1}{2}, \frac{1}{2}\right]}\left(f_{1}\right) I_{\left[-\frac{1}{2}, \frac{1}{2}\right]}\left(f_{2}\right) \\
\\
\times \frac{I_{\left\{1+\left(-h_{3}\right)^{+}, \ldots, N-1-\left(h_{3}\right)^{+}\right\}}(k)}{N-1} .
\end{aligned}
$$

Furthermore, similar manipulations to those in (11), (12), and (14) lead to

$$
\begin{aligned}
\int_{\Omega} \sqrt{p\left(\mathbf{y} \mid \boldsymbol{\theta}+\mathbf{h}_{3}\right) p(\mathbf{y} \mid \boldsymbol{\theta})} \mathrm{d} \mathbf{y}= \\
\exp \left\{-\frac{1}{2 \sigma^{2}}\left(\left|h_{3}\right|-\sum_{n=k+1}^{k+\left|h_{3}\right|} \cos \left(2 \pi\left(f_{2}-f_{1}\right) n\right)\right)\right\}
\end{aligned}
$$

The expression of $\zeta\left(\mathbf{h}_{3}, \mathbf{0}\right)$ is then obtained by integrating out $f_{1}$ and $f_{2}$ and summing over $\mathbb{N}$ :

$$
\begin{aligned}
\zeta\left(\mathbf{h}_{3}, \mathbf{0}\right)=\frac{\exp \left\{-\frac{\left|h_{3}\right|}{2 \sigma^{2}}\right\}}{(N-1)} \int_{-\frac{1}{2}}^{\frac{1}{2}} \int_{-\frac{1}{2}}^{\frac{1}{2}} \sum_{k=1}^{N-1-\left|h_{3}\right|} \exp \left\{\frac{1}{2 \sigma^{2}}\right. \\
\left.\times \sum_{n=k+1}^{k+\left|h_{3}\right|} \cos \left(2 \pi\left(f_{2}-f_{1}\right) n\right)\right\} \mathrm{d} f_{1} \mathrm{~d} f_{2}
\end{aligned}
$$

which can be efficiently numerically evaluated [19]. Finally, in the same manner as in (18) and (19), we obtain

$$
G_{3,3}=\frac{2\left(1-\zeta\left(2 \mathbf{h}_{3}, \mathbf{0}\right)\right)}{\zeta^{2}\left(\mathbf{h}_{3}, \mathbf{0}\right)}
$$

whose explicit expression is deduced from (23).

\subsubsection{Cross-terms derivation}

Similar considerations to those in subsection 3.2.1 lead to

$$
\begin{aligned}
& \zeta\left(\mathbf{h}_{1}, \mathbf{h}_{2}\right)= \frac{\left(1-\left|h_{1}\right|\right)\left(1-\left|h_{2}\right|\right)}{N-1} \\
& \times \sum_{k=1}^{N-1} \exp \left\{-\frac{1}{2 \sigma^{2}}\left(N-\cos \left(\pi h_{1}(k+1)\right) \frac{\sin \left(\pi h_{1} k\right)}{\sin \left(\pi h_{1}\right)}-\right.\right. \\
&\left.\left.\quad \cos \left(\pi h_{2}(N+k+1)\right) \frac{\sin \left(\pi h_{2}(N-k)\right)}{\sin \left(\pi h_{2}\right)}\right)\right\}
\end{aligned}
$$

which directly implies, by (6), that $G_{1,2}=G_{2,1}=0$.

So far, two terms remain to be derived: $G_{1,3}$ and $G_{2,3}$. They are actually very similar, so details are given only for the former. The denominator terms of $G_{1,3}$ are given by (15) and (23). We then derive $\zeta\left(\mathbf{h}_{1}, \mathbf{h}_{3}\right)$ and we obtain

$$
\begin{aligned}
& \zeta\left(\mathbf{h}_{1}, \mathbf{h}_{3}\right)=\int_{\Theta}\left[\int_{\Omega} \sqrt{p\left(\mathbf{y} \mid \boldsymbol{\theta}+\mathbf{h}_{1}-\mathbf{h}_{3}\right) p(\mathbf{y} \mid \boldsymbol{\theta})} \mathrm{d} \mathbf{y}\right] \\
& \times \sqrt{p\left(\boldsymbol{\theta}+\mathbf{h}_{1}-\mathbf{h}_{3}\right) p(\boldsymbol{\theta})} \mathrm{d} \boldsymbol{\theta}
\end{aligned}
$$

and similarly as in (10) and (21), we have

$$
\begin{aligned}
\sqrt{p\left(\boldsymbol{\theta}+\mathbf{h}_{1}-\mathbf{h}_{3}\right) p(\boldsymbol{\theta})} & =I_{\left[-\frac{1}{2}+\left(-h_{1}\right)^{+}, \frac{1}{2}-\left(h_{1}\right)^{+}\right]}\left(f_{1}\right) \\
\times I_{\left[-\frac{1}{2}, \frac{1}{2}\right]}\left(f_{2}\right) & \frac{I_{\left\{1+\left(h_{3}\right)^{+}, \ldots, N-1-\left(-h_{3}\right)^{+}\right\}}(k)}{N-1} .
\end{aligned}
$$


Finally, since

$$
\begin{aligned}
\int_{\Omega} \sqrt{p\left(\mathbf{y} \mid \boldsymbol{\theta}+\mathbf{h}_{1}-\mathbf{h}_{3}\right) p(\mathbf{y} \mid \boldsymbol{\theta})} \mathrm{d} \mathbf{y}= \\
\exp \left\{-\frac{1}{2 \sigma^{2}}\left[k+\left|h_{3}\right|-\sum_{n=1}^{k} \cos \left(2 \pi h_{1} n\right)-\right.\right. \\
\left.\left.\sum_{n=k+1}^{k+\left|h_{3}\right|} \cos \left(2 \pi\left(f_{2}-f_{1}-h_{1} I_{\mathbb{R}_{-}^{*}}\left(h_{3}\right)\right) n\right)\right]\right\}
\end{aligned}
$$

where $\left.\mathbb{R}_{-}^{*} \triangleq\right]-\infty, 0[$, we are now able to write the expression of $\zeta\left(\mathbf{h}_{1}, \mathbf{h}_{3}\right)$ by substituting (27) and (28) into (26), which leads to

$$
\begin{aligned}
& \zeta\left(\mathbf{h}_{1}, \mathbf{h}_{3}\right)=\frac{1}{N-1} \exp \left\{-\frac{\left|h_{3}\right|}{2 \sigma^{2}}\right\} \times \\
& \int_{-\frac{1}{2}}^{\frac{1}{2}} \int_{-\frac{1}{2}+\left(-h_{1}\right)}^{\frac{1}{2}-\left(h_{1}\right)^{+}} \sum_{k=1}^{N-1-\left|h_{3}\right|} \exp \left\{-\frac{1}{2 \sigma^{2}}\left[k-\sum_{n=1}^{k} \cos \left(2 \pi h_{1} n\right)-\right.\right. \\
& \left.\left.\sum_{n=k+1}^{k+\left|h_{3}\right|} \cos \left(2 \pi\left(f_{2}-f_{1}-h_{1} I_{\mathbb{R}_{-}^{*}}\left(h_{3}\right)\right) n\right)\right]\right\} \mathrm{d} f_{1} \mathrm{~d} f_{2},(29)
\end{aligned}
$$

and from which the expressions of $\zeta\left(-\mathbf{h}_{1},-\mathbf{h}_{3}\right), \zeta\left(\mathbf{h}_{1},-\mathbf{h}_{3}\right)$, and $\zeta\left(-\mathbf{h}_{1}, \mathbf{h}_{3}\right)$ are obtained. Then one can deduce $G_{1,3}$ from (6). Notice that generally, $G_{1,3} \neq 0$.

Finally, the expression of $\zeta\left(\mathbf{h}_{2}, \mathbf{h}_{3}\right)$ is obtained in the same way as that of $\zeta\left(\mathbf{h}_{1}, \mathbf{h}_{3}\right)$ as

$$
\begin{aligned}
& \zeta\left(\mathbf{h}_{2}, \mathbf{h}_{3}\right)=\frac{1}{N-1} \exp \left\{-\frac{N}{2 \sigma^{2}}\right\} \times \\
& \int_{-\frac{1}{2}+\left(-h_{2}\right)^{+}}^{\frac{1}{2}-\left(h_{2}\right)^{+}} \int_{-\frac{1}{2}}^{\frac{1}{2}} \sum_{k=1}^{N-1-\left|h_{3}\right|} \exp \left\{\frac { 1 } { 2 \sigma ^ { 2 } } \left[\begin{array}{c}
k+\sum_{n=k+\left|h_{3}\right|+1}^{N} \cos \left(2 \pi h_{2} n\right)+ \\
\left.\left.\sum_{n=k+1}^{k+\left|h_{3}\right|} \cos \left(2 \pi\left(f_{2}+h_{2} I_{\mathbb{R}_{+}^{*}}\left(h_{3}\right)-f_{1}\right) n\right)\right]\right\} \mathrm{d} f_{1} \mathrm{~d} f_{2},
\end{array}\right.\right.
\end{aligned}
$$

where $\left.\mathbb{R}_{+}^{*} \triangleq\right] 0,+\infty[$.

As for $G_{1,3}$, the expression of $G_{2,3}$ arises from (20), (23) and (30), that one properly substitutes into (6).

\section{NUMERICAL RESULTS}

In this section, we present the simulation results comparing the empirical global mean square errors (GMSE) of the maximum a posteriori (MAP) estimator of $\left(f_{1}, f_{2}, \tau\right)$ with the WWB derived above, for model (2) with $N=20$. Notice that the maximization with respect to the $h_{q}, q=1,2,3$ is performed by maximizing the trace of $\mathbf{H G}^{-1} \mathbf{H}^{T}$.

In Figs. 1 and 2, we perform 1000 Monte-Carlo simulations. These figures reveals that, as expected, the WWB in good agreement with the behavior of the MAP estimator and remains reasonably tight. Indeed, Fig. 1 which represents the GMSE of the MAP and WWB for the change-point $\tau$ shows a gap of approximately 1 sample at low and high SNR.

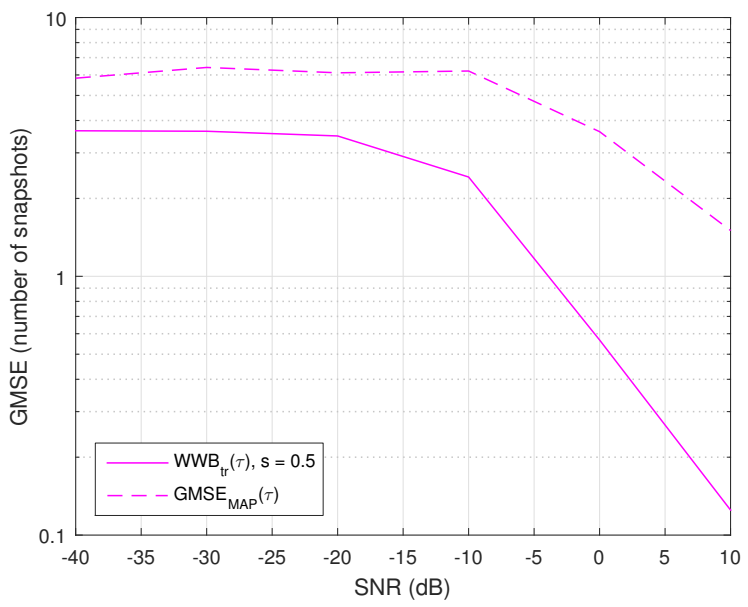

Fig. 1. GMSE of the MAP estimator (dashed line) and WWB (solid line) for the estimation of the change-point $\tau$ (in magenta), with $N=20$ snapshots.

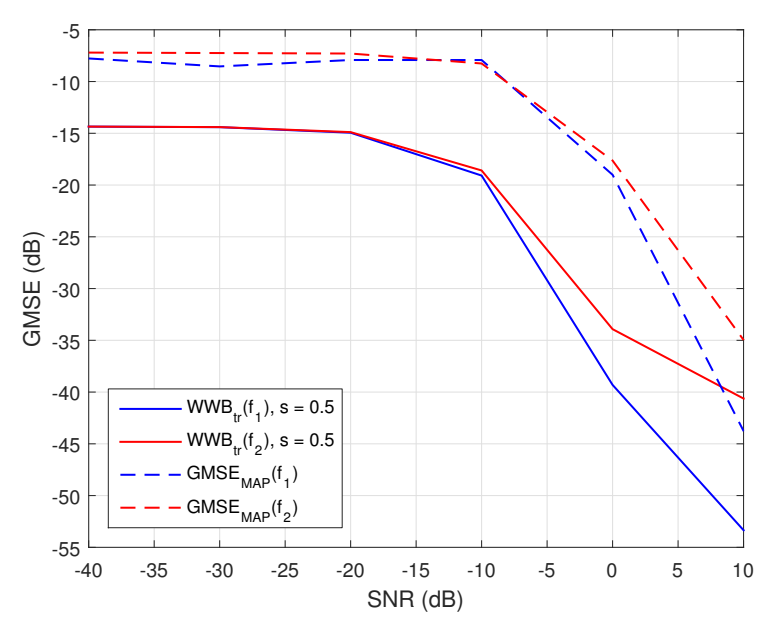

Fig. 2. GMSE of the MAP estimator (dashed lines) and WWB (solid lines) for the estimation of the frequencies $f_{1}$ (in blue) and $f_{2}$ (in red), with $N=20$ snapshots.

\section{CONCLUSION}

In this communication, we derived the Weiss-Weinstein bound (WWB) in the case of an abruptly changing frequency. Numerical simulations reveal that the WWB with respect to the frequencies and the change-point remains reasonably tight regarding to the global mean square error of the maximum a posteriori estimator.

\section{REFERENCES}

[1] M. Basseville and I. V. Nikiforov, Detection of Abrupt Changes, Theory and Application., Englewood Cliffs, NJ: Prentice-Hall, 1993. 
[2] J.-Y. Tourneret, M. Doisy, and M. Lavielle, "Bayesian off-line detection of multiple change-points corrupted by multiplicative noise: application to SAR image edge detection," ELSEVIER Signal Processing, vol. 83, no. 9, pp. 1871-1887, Sept. 2003.

[3] N. Brunel and F. Barbaresco, "Doppler and Polarimetric Statistical Segmentation for Radar Clutter map based on Pairwise Markov Chains," in Proc. of IEEE RADAR. Oct. 2007.

[4] N. A. Nechval, K. N. Nechval, and E. Vasermanis, "Detection of target signals in clutter using change point statistics," in Proc. SPIE 4541, Image and Signal Processing for Remote Sensing VII, 111, Jan. 2002.

[5] D. V. Hinkley, "Inference about the change-point in a sequence of random variables," Biometrika, vol. 57, no. 1, pp. 1-18, 1970.

[6] S. B. Fotopoulos and S. K. Jandhyala, "Maximum likelihood estimation of a change-point for exponentially distributed random variables," ELSEVIER Statistics and Probability Letters, vol. 51, pp. 423-429, 2001.

[7] S. B. Fotopoulos, S. K. Jandhyala, and E. Kapalova, "Exact asymptotic distribution of change-point MLE for change in the mean of Gaussian sequences," The Annals of Applied Statistics, vol. 4, no. 2, pp. 1081-1104, Nov. 2010.

[8] H. Cramér, Mathematical Methods of Statistics, vol. 9 of Princeton Mathematics, Princeton University Press, New-York, Sept. 1946.

[9] E. L. Lehmann and G. Casella, Theory of Point Estimation, Springer Texts in Statistics. Springer, NewYork, NY, USA, 2 edition, Sept. 2003.

[10] A. Bartov and H. Messer, "Analysis of inherent limitations in localizing step-like singularities in a continuous signal," in Proc. IEEE SP Int. Symp. Time-Frequency Time-Scale Analysis, Paris, France, June 1996, pp. 2124.

[11] A. Swami and B. Sadler, "Cramér-Rao bounds for stepchange localization in additive and multiplicative noise," in Proc. of IEEE Workshop on Statistical Signal and Array Processing (SSAP), Portland, OR, USA, Sept. 1998, pp. 403-406.

[12] A. Ferrari and J.-Y. Tourneret, "Barankin lower bound for change points in independent sequences," in Proc. of IEEE Workshop on Statistical Signal Processing (SSP), St. Louis, MO, USA, Sept. 2003, pp. 557-560.

[13] P. S. La Rosa, A. Renaux, A. Nehorai, and C. H. Muravchik, "Barankin-type lower bound on multiple change-point estimation," IEEE Transactions on Signal Processing, vol. 58, no. 11, pp. 5534-5549, Nov. 2010.

[14] L. Bacharach, A. Renaux, M. N. El Korso, and E. Chaumette, "Weiss-Weinstein bound for changepoint estimation," in Proc. of IEEE International Workshop on Computational Advances in Multi-Sensor Adaptive Processing (CAMSAP), Cancún, Mexico, Dec. 2015, pp. 477-480.

[15] D. Ramakrishnan and J. Krolik, "Target detection in abruptly non-stationary Doppler-spread clutter," in Proc. of IEEE International Conference on Acoustics, Speech, and Signal Processing (ICASSP), Toulouse, France, May 2006, vol. 3, pp. 185-188.

[16] D. C. Rife and R. R. Boorstyn, "Single tone parameter estimation from discrete time observations," IEEE Transactions on Information Theory, vol. 20, no. 5, pp. 591-598, Sept. 1974.

[17] H. L. Van Trees and K. L. Bell, Eds., Bayesian Bounds for Parameter Estimation and Nonlinear Filtering/Tracking, Wiley/IEEE Press, New-York, NY, USA, Sept. 2007.

[18] E. Weinstein and A. J. Weiss, "A general class of lower bounds in parameter estimation," IEEE Transactions on Information Theory, vol. 34, no. 2, pp. 338-342, Mar. 1988.

[19] L. F. Shampine, "MATLAB Program for Quadrature in 2D," Applied Mathematics and Computation, vol. 202, no. 1, pp. 266-274, Aug. 2008. 